\title{
The role of using ultra short pulsed lasers in determining dynamical dependent of free electron density in the skin tissue for treatment of skin cancer
}

\author{
S. Nasrin Hosseinimotlagh, Samira Khazraei \\ Department of Physics, Shiraz branch Islamic Azad University, Shiraz, Iran \\ Email address: \\ hoseinimotlagh@hotmail.com (S. N. Hosseinimotlagh), Sarniraa@gmail.com (S. Khazraei)
}

To cite this article:

S. Nasrin Hosseinimotlagh, Samira Khazraei. The Role of Using Ultra Short Pulsed Lasers in Determining Dynamical Dependent of Free Electron Density in the Skin Tissue for Treatment of Skin Cancer. Cancer Research Journal. Vol. 2, No. 2, 2014, pp. 33-41.

doi: $10.11648 /$ j.crj.20140202.14

\begin{abstract}
We study on the new advances in laser cell surgery for skin cancer treatment and investigate on the working mechanisms of femtosecondlaser. Also, influences of the critical electron density and radiation intensity on the freeelectron density for ablation on the epidermis and dermis tissues are investigated. Our studies on this work, show that if the amount of radiation intensity of pulsed laser is increased then the distance of focal spot is decreased also the amount of time dependent free- electron density become more and more, such that this case is proper for skin cancer treatment. Also, our calculations for skin tissue show that optimum time dependent free electron density for dermis layer at angle $16^{\circ}$ and $22^{\circ}$ which is a function of wavelength, beam width, beam radius, amplitude of the beam radiation strength and pulse duration is more than for epidermis layer.
\end{abstract}

Keywords: Skin, Tissue, Plasma, Ultra-Short

\section{Introduction}

The great progress in laser treatment in clinical applications is primarily attributed to the rapid development of pulsed laser systems. The use of highly focused pulsed laser beam to modify and manipulate tissue medium for applications in molecular and cellular biophysics and biotechnology becomes more pervasive $[1,2]$. Ultrafast lasers output ultra-short pulses with pulse widths ranging from picoseconds down to femtoseconds. Short-pulse lasers have become greatly important in recent years for many technologies, including laser cleaning, and medical and biological applications. Applications include dissection or inactivation of cellular organelles and chromosomes with submicron spatial precision [3], and gene delivery through transient membrane disruption [4], to name a few. As the pulsed laser micro beam technologies continue to advance, the fundamental knowledge of lasertissue interaction mechanisms is of vital importance and could provide a framework where in systematic investigation of novel pulsed micro beam techniques can be developed. The plasma-mediated ablation model has been employed to explain the optical breakdown phenomenon in the visible and near-infrared wavelength region, in which plasma induced by the strong electromagnetic field of a short laser pulse without significant light absorption, is considered as the cause of breakdown [1, 5-6].During optical breakdown, a very high density of free electrons, i.e., a plasma, of the order of $10^{-40}-10^{27} \mathrm{~cm}^{-3}$, is produced. The mechanism, chromophor ionization, is responsible for laser-induced optical breakdown, which results in a geometric increase in the free-electron density.

Plasma formation is characterized by the growth of plasmas. Docchio et al. [7, 8] estaimated the "moving breakdown" model to analyze plasma starting times and the temporal and spatial dynamics of plasma expansion in liquids subject to nanosecond laser pulse irradiation. Fan et al. [10] modified the model by taking into account the pulse propagation to characterize the time and space dependent breakdown region induced by femtosecond laser pulses. Recently, Zhou et al. [11] used the rate equation to investigate the kinetic progression of plasma in pure water generated by focused short laser pulses. Recently, Guo's group investigated plasma-induced ablation [12, 13] as well as thermal interaction [14] of focused short laser pulses with skin tissues.

It is now acknowledged that pulses with very short durations, such as picosecond or femtosecond, are 
advantageous in many applications. Due to the extremely short pulse duration which is much shorter than the thermal relaxation time of many materials, laser interaction with materials occurs before heat diffusion ever takes place, leading to increased local temperature rise in a very short time period and minimization of heat-affecting zone. This will lessen thermal damage to the surroundings. Then the ablation of skin tissue with pico and femtosecond laser pulses is investigated. In this paper, we discussed the interaction fundamentals between ultra short laser with skin tissue. The model skin tissue is an axisymmetric cylinder and are stratified as three layers with different optical properties similar to human skin tissues-epidermis, dermis, and subcutaneous fat, respectively. The ultra short laser pulse is converged inside the skin tissue to induce plasma breakdown for ablating cancerous tissue. The intensity of the focused beam is calculated by the Beer-Lambert law, and the time-dependent freeelectron density is obtained according to fourth-order Runge-Kutta method. Various ionization mechanism such as chromophore is calculated to show effects on the tissue layers. In this model, the temporal evolution of free electrons (i.e. plasmas) is predicted by a rate equation. The temporal evolution of the free-electron density is also illustrated at different locations inside the skin tissue. Finally, we conclude that of the influences of the free electron density rate equation and the chromophore ionization rate, beam radius, amplitude of the beam radiation strength on the ablation in human skin.

\section{Mathematical Models}

$$
I^{b}(r, z, t)=I_{0}(z) \exp \left\{-4 \ln 2\left[\frac{\left(t-\frac{\left|z-z_{0}\right|}{c \cos \theta}\right)}{t_{p}}-2\right]^{2}\right\} \exp \left[\frac{-2 r^{2}}{\omega(z)^{2}}\right] \exp \left(-\frac{\sigma_{e}\left|z-z_{0}\right|}{\cos \theta}\right)
$$

Where $c$ is the speed of light in the tissue; $\theta$ is the angle between the incident laser and the optical axis; $t_{p}$ is the pulse width at half maximum; $\sigma_{e}$ is the extinction coefficient, which is the summation of the absorption coefficient $\sigma_{a}$ and the scattering coefficient $\sigma_{s}$. The total time duration of a whole pulse is set as $4 t \mathrm{p}$ in this study, so that the peak of the pulse arrives at time $2 \mathrm{tp}$. In our calculations we assumed that average laser power is $0.065 \mathrm{~W}$. The amplitude of the beam radiation strength $I_{0}(Z)$ can be specified by equation following

$$
I_{0}(Z)=\frac{2 P}{\pi \omega(z)^{2}}
$$

Beam spot size $\omega$ is a function of axial position $z$, and it is characterized by beam waist $\omega_{0}$ at the focal point and at the Rayleigh range or focal region $Z_{R}$ as following [14]:

\subsection{Time-Dependent Equation of Radiative Transfer (ERT)}

Consider a converging short laser pulse upon a biological tissue cylinder is sketched in Figure (1).[14]. The laser beam radiation heat transfer is described by the time-dependent ERT in the cylindrical coordinate system as where $\mu_{l}, \eta_{l}, \xi_{l}$ are the three directional cosines for the discrete direction $\hat{s}^{l}$ is the radiative source term (the superscript $l$ is the index of discrete directions), $\mathrm{c}$ is the speed of light in tissue medium, I is the radiation intensity, $\sigma_{e}$ is the extinction coefficient that is the sum of the absorption coefficient $\sigma_{a}$ and the scattering coefficient $\sigma_{s}, \mathrm{t}$ is the time[16]

$$
\begin{gathered}
\frac{1}{C} \frac{\partial I_{l}^{d}}{\partial t}+\frac{\mu_{l}}{r} \frac{\partial}{\partial r}\left(r I_{l}^{d}\right)-\frac{1}{r} \frac{\partial}{\partial \varphi}\left(\eta_{l} I_{l}^{d}\right)+\xi_{l} \frac{\partial I_{l}^{d}}{\partial z}+\sigma_{e} I_{l}^{d}=\sigma_{e} S_{l} l= \\
1,2, \ldots, N
\end{gathered}
$$

Once the intensity field is obtained, the incident radiation is calculated as $[16,17]$

$$
G=\sum_{l=1}^{N} \omega_{l} I_{l}^{d}+I^{b}
$$

The incident radiation is the total intensity impinging on a point from all directions. For an extremely weakly scattering medium like skin tissue, the value of the incident radiation equals to the ballistic laser irradiance $I^{b}$. The radiation intensity has two components: the ballistic component $I^{b}$ accounting for the incident laser beam and the diffuse component $I^{d}$ standing for the scattering component of the laser beam. The ballistic component of a laser beam is expressed as [15-16]

$$
\omega(Z)=\omega_{0 R}\left[1+\left(\frac{Z}{z_{R}}\right)^{2}\right]^{\frac{1}{2}}
$$

Where $\omega_{0 R}$ is the beam waist at the focus; and $Z_{R}$ is Rayleigh length that is calculated by

$$
Z_{R}=\frac{n \pi \omega_{0 R}{ }^{2}}{\lambda M^{2}}
$$

Where, $\mathrm{n}$ is the refractive index of the medium; $\lambda$ the laser wavelength in free space; $M^{2}$ is the beam quality factor that is expressed as

$$
M^{2}=\frac{\omega_{0 R} \theta_{R}}{\theta \omega_{0}}
$$

Here, $\theta_{R}$ is the far-field divergence of a real beam; $\omega_{0}$ and $\theta$ arethe beam waist and far-field divergence of a true Gaussian beam, respectively. In our calculations we assume that $M^{2}=1$ for skin tissue. 


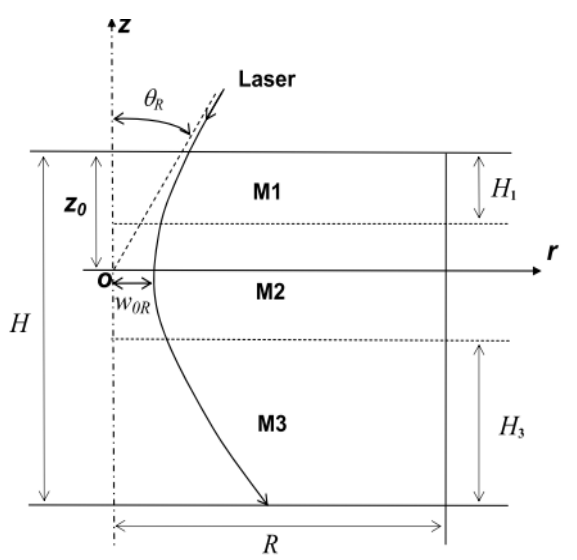

Figure 1.Sketch of the geometrical coordinates and dimension[14].

\subsection{Rate Equation}

The general form of the rate equation of the time evolution of the free electron density is given by [17].

$$
\frac{\partial \rho}{\partial t}=\left(\frac{\partial \rho}{\partial t}\right)_{m p}+\left(\frac{\partial \rho}{\partial t}\right)_{c h}+\eta_{c a s c} \rho-g \rho-\eta_{r e c} \rho^{2}
$$

Where $\rho$ is the time dependent free-electron density. The first three terms on the right hand side represent the production of free electrons through multi photon, chromophore and cascade (avalanch) ionizations, respectively. Last two terms are the electron losses due to diffusion and recombination, respectively. The multi photon and cascade (avalanch) ionizations and diffusion rates are expressed as [14]

$$
\left(\frac{\partial \rho}{\partial t}\right)_{m p} \approx \frac{2 \omega}{9 \pi}\left(\frac{m^{\prime} \omega}{\hbar}\right)^{\frac{3}{2}}\left[\frac{e^{2}}{16 m^{\prime} \Delta E \omega^{2} c n \varepsilon_{0}} G\right]^{k} \exp (2 k) \varphi\left(\sqrt{2 k-\frac{2 \Delta E}{\hbar \omega}}\right)
$$

$$
\begin{aligned}
& \eta_{\text {casc }}=\frac{1}{\omega^{2} \tau^{2}+1}\left(\frac{e^{2} \tau}{c n m \Delta E \varepsilon_{0}} G-\frac{m \tau \omega^{2}}{M_{m}}\right) \\
& g=\frac{\tau \Delta E}{3 m}\left[\left(\frac{2.4}{\omega_{0 R}}\right)^{2}+\left(\frac{1}{Z_{R}}\right)^{2}\right]
\end{aligned}
$$

$\eta_{\text {rec }}=2 \times 10^{-9} \mathrm{~cm}^{3} / \mathrm{s}[18]$. The ionization energy is $E=6.5$ $\mathrm{eV}$ and $E=4.6 \mathrm{eV}$ for water medium [19] and skin tissue [20], respectively. The second term on the right hand side eq.(5), the quasi-free-electron density due to chromophore ionization is estimated as [20]

The recombination rate is considered to be

$$
\rho_{c h}(t)=\frac{3 \sqrt{\pi}}{4} \eta_{c h} N_{b}\left[\frac{2 k_{B}}{\Delta E}\left(T_{0}+\frac{F(t) \mu_{a}}{c_{c h} u_{c h} f_{c h}}\right)\right]^{1 / 2} \exp \left\{-\frac{\Delta E}{2 k_{B}\left[T_{0}+\frac{F(t) \mu_{a}}{c_{c h} u_{c h} f_{c h}}\right]}\right\}
$$

A necessary condition for the chromophore ionization pathway in producing quasi-free electrons lie in adiabatic heating of the chromophores by the ultra short laser pulses. The characteristics time of thermal energy transport out of an described chromophore is proposed to be $20 \mu$ s [20]. Therefor, the adiabatic heating condition can be assumed for the ablation induced duo to picosecond or femtosecond laser interaction within each medium. Where $F(t)=$ $\int_{0}^{t} G(t) \mathrm{dt}$ is the time dependent integral of the incident radiation: $\eta_{c h} \sim 1 \times 10^{15} \mathrm{~m}^{-3}$ is the number density of chromophores in skin tissue, $N_{b} \approx 1 \times 10^{11}$ is the average number of bound electrons per chromophore, $u_{c h}=1.35 \times$ $10^{3} \frac{\mathrm{kg}}{\mathrm{m}^{3}}$ mass density, $k_{B}$ is the Boltzmann constant; $T_{0}$ is the ambient temperature assumed to be $300 \mathrm{~K}, c_{c h}=$ $2.51 \times 10^{3} \frac{\mathrm{J}}{\mathrm{kgK}}$ is the specific heat of melanosome organelles, $f_{c h} \approx 0.5 \%$ is the volume ratio of the chromophores to skin [20]. By taking a partial derivative of the free-electron density with respect to time, the chromophore ionization rate is calculated.

\subsection{Properties of Skin Tissue}

In this paper, for comparing our obtained results of skin tissue, we are considered the common wavelengths 532, 580 and $1064 \mathrm{~nm}$. A constant absorption and scattering coefficients of the plasmas generated by the pulsed laser is assumed. The epidermis layer absorption coefficient is adopted as $\sigma_{\text {aed }}=0.02 \mathrm{~mm}^{-1}$ and for dermis is $\sigma_{a d}=$ $0.05 \mathrm{~mm}^{-1}$, the optical properties of the tissues for medium at three wavelength are listed in Table 1.In this work, human skin $(\mathrm{H}=5 \mathrm{~mm}, \mathrm{R}=5 \mathrm{~mm})$ is organized in distinct layers and cylindrical coordinate variables are $\mathrm{z}$ and $\mathrm{r}$. Which the high of epidermis, dermis and subcutaneous fat are, $\left(\mathrm{H}_{\mathrm{e}}=0.5 \mathrm{~mm}\right),\left(\mathrm{H}_{\mathrm{d}}=1.5 \mathrm{~mm}\right),\left(\mathrm{H}_{\mathrm{f}}=3 \mathrm{~mm}\right)$, respectively. In this paper, the focused laser beam will incident on the surface of the epidermis and dermis layers within skin tissue. To induce plasma ablation in medium, use of ultra short pulsed (USP) lasers with pulse duration down to picoseconds or femtoseconds is required. The optical properties of the skin tissues at different wavelengths are listed in Table 1. The refractive index of the skin tissues is assumed to be constant at 1.40 [21].

Table 1. Optical properties of the skin tissues at wavelength 1064nm [21].

\begin{tabular}{cccc}
\hline Tissue type & Epidermis & Dermis & Fat \\
\hline$\sigma_{a}\left(\mathrm{~mm}^{-1}\right)$ & 0.02 & 0.05 & 0.07 \\
$\sigma_{s}\left(\mathrm{~mm}^{-1}\right)$ & 3.0 & 1.83 & 1.69 \\
\hline
\end{tabular}

\section{Results and Discussion}

Using the presented models and available data, we consider the time dependent free electron density in skin tissue. There for, a laser beam is assumed to be converged to a small spot at the skin layer by a single objective lens with angle of 16 and 22 degree. Now the study on the plasma-mediated ablation is extended to the model and skin tissue for different layers. A focused laser beam will incident on the surface of the epidermis or inside (dermis) in skin tissue. The focused laser beam technology is 
adopted to its propagation in the skin tissue is simulated via solving the kinetic equation of radiative transfer with RungKutta method. In the numerical model, the optical breakdown in the skin tissue is identified when the maximum free- electron density generated at the focal spot center during the period of one laser pulse equals to a critical electron density. In this work, we calculated the time evolution of the free-electron density in a control volume. The technique of converging beam is a non-

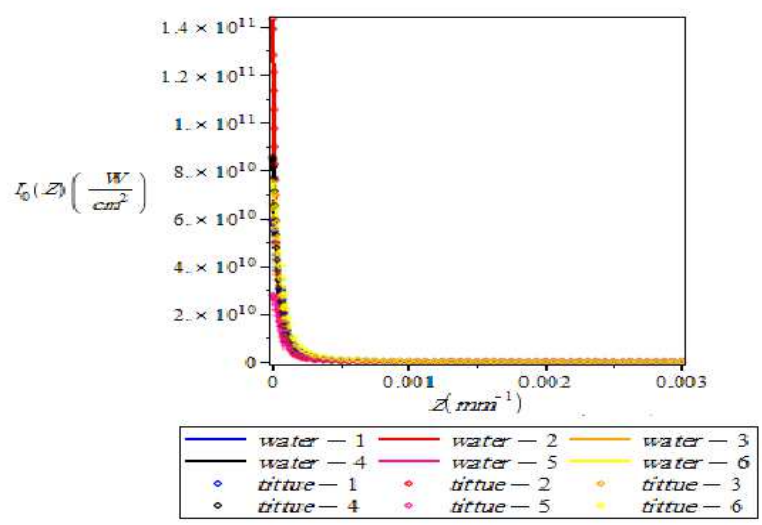

a invasive method which ideally delivers energy to a location inside the body and avoids undesirable reactions in the surrounding healthy tissues. To study on the ablation in a biological tissue, the attenuation of lights due to the absorption and scattering effects are such that needs to be accurately taken into account. Our numerical calculations for free electron density at three wavelength show that, the highest free-electron density would be occured in the layer dermis at 16 degree.

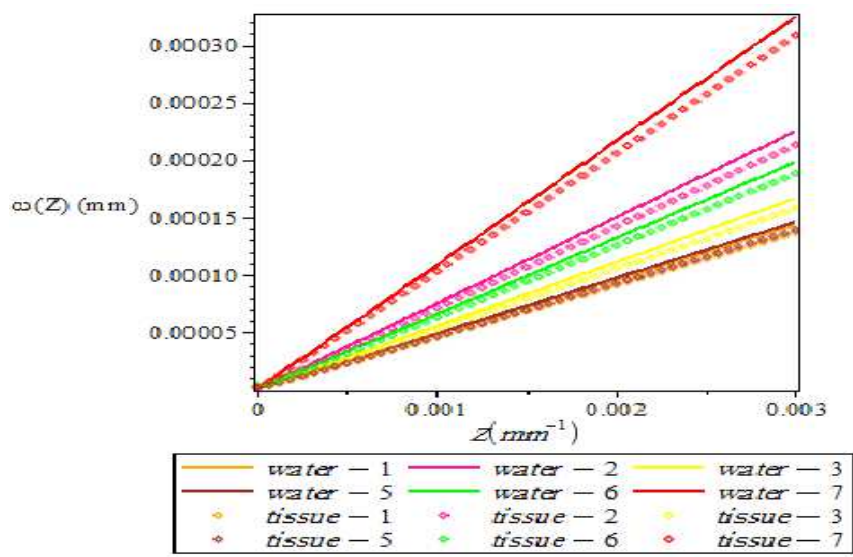

$\mathrm{b}$

Figure 2.Comparison of the skin tissue and water medium a) $\omega(z)$ b) $I_{0}(z)$ as a functions of the $Z\left(\mathrm{~mm}^{-1}\right)$.

Table 2.Comparison $\omega(z)$ and $I_{0}(z)$ calculated in the skin tissue water medium.

\begin{tabular}{|c|c|c|c|c|c|c|c|c|c|c|c|}
\hline & $\lambda(n m)$ & $I_{0 s}(Z)$ & $2 \omega_{0 R}(\mu m)$ & $\omega_{z s}\left(\begin{array}{c}0.001 \\
m^{-1}\end{array}\right)$ & $\omega_{z s}\left(\begin{array}{l}0.002 \\
m^{-1}\end{array}\right)$ & $\omega_{z s}\left(\begin{array}{l}0.003 \\
m^{-1}\end{array}\right)$ & $t_{p}(p s)$ & $I_{0 w}(Z)$ & $\omega_{z w}\left(\begin{array}{l}0.001 \\
m^{-1}\end{array}\right)$ & $\omega_{z w}\left(\begin{array}{l}0.002 \\
m m^{-1}\end{array}\right)$ & $\omega_{z w}\left(\begin{array}{l}0.003 \\
m m^{-1}\end{array}\right)$ \\
\hline 1 & 532 & $2.06 \times 10^{8}$ & 5.3 & 0.0000459 & 0.0000916 & 0.000137 & 6000 & $2.02 \times 10^{8}$ & 0.0000487 & 0.0000964 & 0.000144 \\
\hline 2 & 532 & $4.52 \times 10^{8}$ & 3.4 & 0.0000719 & 0.000143 & 0.000213 & 30 & $4.51 \times 10^{8}$ & 0.0000756 & 0.000151 & 0.0000224 \\
\hline 3 & 580 & $2.21 \times 10^{8}$ & 5 & 0.0000531 & 0.000106 & 0.000158 & 3 & $2.2 \times 10^{8}$ & 0.0000558 & 0.000111 & 0.000166 \\
\hline 4 & 580 & $2.21 \times 10^{8}$ & 5 & 0.0000531 & 0.000106 & 0.000158 & 0.3 & $2.2 \times 10^{8}$ & 0.0000558 & 0.000111 & 0.000166 \\
\hline 5 & 580 & $2.77 \times 10^{8}$ & 4.4 & 0.0000468 & 0.0000934 & 0.000139 & 0.1 & $2.76 \times 10^{8}$ & 0.0000491 & 0.000124 & 0.000147 \\
\hline 6 & 1064 & $9.86 \times 10^{8}$ & 7.7 & 0.0000637 & 0.000127 & 0.000189 & 6000 & $9.69 \times 10^{8}$ & 0.0000639 & 0.000133 & 0.000198 \\
\hline 7 & 1064 & $2.65 \times 10^{8}$ & 4.7 & 0.000104 & 0.000207 & 0.000309 & 30 & $2.6 \times 10^{8}$ & 0.000108 & 0.000218 & 0.000324 \\
\hline
\end{tabular}

Figure 2(a) show the distance variations of $\omega(\mathrm{z})$ (beam radius) eq.5, for various wavelengths such as 532,580,1064 $\mathrm{nm}$ at different physical conditions that are listed in Table 2 for water medium (solid line) and skin tissue (spot line). Here we estimated $\omega(\mathrm{z})$ produced by laser pulse $30 \mathrm{ps}$ at wavelength $1064 \mathrm{~nm}$ is maximum and the laser pulse 6000 ns at wavelength 532 is minimum. We find that $\omega(\mathrm{z})$ in the water medium much is greater than that for skin tissue, due to differences in the refractive index, hence, the Rayleigh length calculated is different. Fig. 2(b) show the distance variations of $I_{0}(z)$ (beam radiation strength) eq.4 for various wavelengths at different physical conditions that for water medium(solid line) and skin tissue (spot line). $I_{0}(z)$ calculated in the skin tissue is more than water medium, duo to differences in the refractive index and the Rayleigh length calculated, then maximum calculate $I_{0}(z)$ from the focus spot the incoming laser beam $(\mathrm{z}=0)$. The maximum irradiance is always at the beam focus [14]. The extinction coefficient is from the layers dermis
$1.88 \mathrm{~mm}^{-1}$ and epidermis $3.02 \mathrm{~mm}^{-1}$

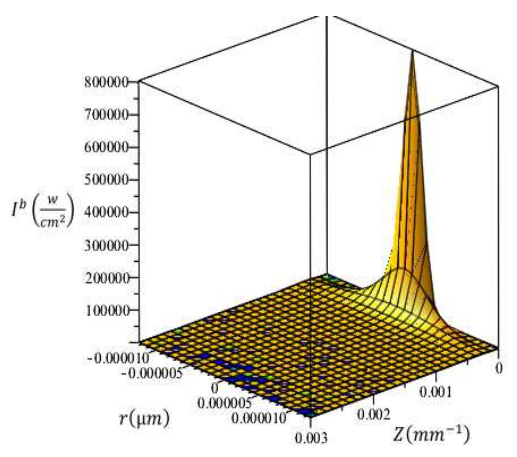

tissue derm 16 tissue derm 22 tissue epiderm 16 tissue epiderm 22

Figure 3. Comparison of $I^{b}\left(\frac{w}{\mathrm{~cm}^{2}}\right)$ as a function of the $r(\mu \mathrm{m})$ and $Z\left(\mathrm{~mm}^{-1}\right)$ in the wavelength $532 \mathrm{~nm}$ for $2 \omega_{0 R}=3.4 \mu \mathrm{m}$ and $2 \omega_{0 R}=5.3 \mu \mathrm{m}$ at two angles $16^{\circ}, 22^{\circ}$ for dermis and epidermis layers of skin tissue. 


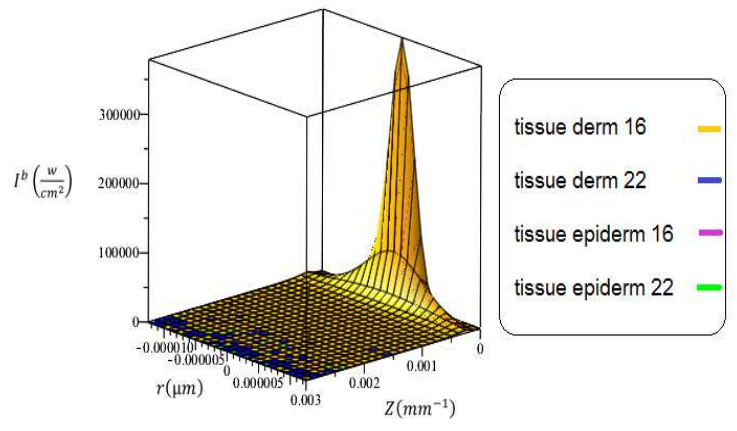

Figure 4. Comparison of $I^{b}\left(\frac{w}{c m^{2}}\right)$ as a function of the $r(\mu \mathrm{m})$ and $Z\left(\mathrm{~mm}^{-1}\right)$ at the wavelength $1064 \mathrm{~nm}$ for $2 \omega_{0 R}=4.7 \mu \mathrm{m}$ and $2 \omega_{0 R}=7.7 \mu \mathrm{m}$ at two angles $16^{\circ}, 22^{\circ}$ for dermis and epidermis layers of skin tissue

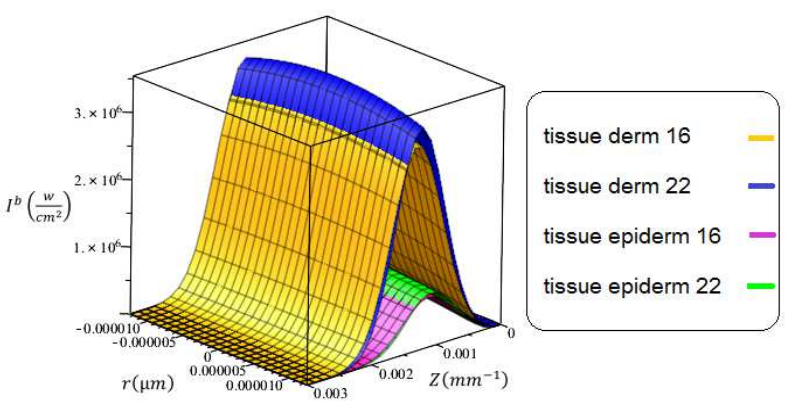

Figure 5. Comparison of $I^{b}\left(\frac{w}{c m^{2}}\right)$ as a function of the $r(\mu \mathrm{m}), Z\left(\mathrm{~mm}^{-1}\right)$ in the wavelength $580 \mathrm{~nm}$ and $2 \omega_{0 R}=5 \mu \mathrm{m}, t_{P}=3$ psat two angles $16^{\circ}, 22^{\circ}$ for dermis and epidermis layers of skin tissue.

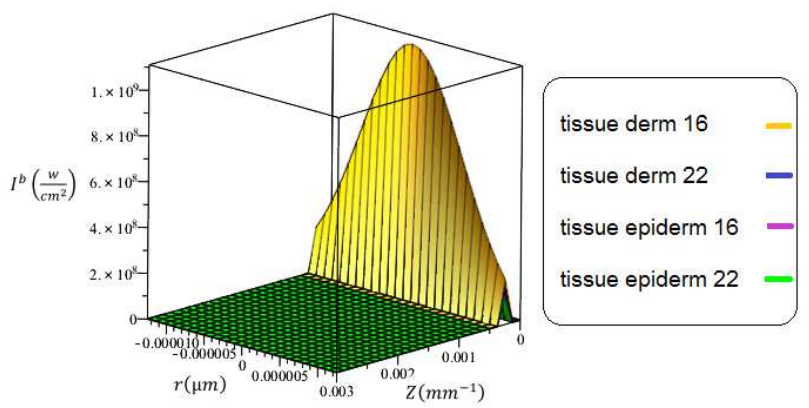

Figure 6. Comparison of $I^{b}\left(\frac{w}{\mathrm{~cm}^{2}}\right)$ as a function of the $r(\mu \mathrm{m}), Z\left(\mathrm{~mm}^{-1}\right)$ in the wavelength $580 \mathrm{~nm}$ and $2 \omega_{0 R}=5 \mu \mathrm{m}, t_{P}=0.3$ psat two angles $16^{\circ}, 22^{\circ}$ for dermis and epidermis layers of skin tissue.

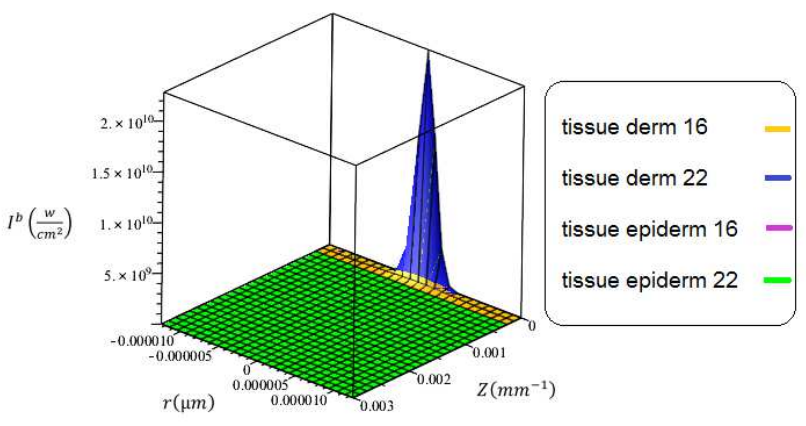

Figure 7. Comparison of $I^{b}\left(\frac{w}{\mathrm{~cm}^{2}}\right)$ in terms of $r(\mu \mathrm{m})$ and $Z\left(\mathrm{~mm}^{-1}\right)$ at the wavelength $580 \mathrm{~nm}$ and $2 \omega_{0 R}=4.4 \mu \mathrm{m}, t_{P}=30$ psat two angles $16^{\circ}, 22^{\circ}$ for dermis and epidermis layers a) Water medium b) Skin tissue.
Figure 5-7 shows intensity radiation laser in the water medium and skin tissue with the $0.3 \mathrm{ps}, 30 \mathrm{ps}$ and 3 ps pulses the layers configuration with considerd amount, angle, pulse duration and the layers depth and distance of focal spot, the first layer is dermis $16^{\circ}$ and second layer is dermis $22^{\circ}$, the $3^{\text {rd }}$ layer is epidermis $16^{\circ}$ and the last layer is epidermis $22^{\circ}$.Also, in fig 3 to 7 ,we will find that the good agreement is seen between intensity radiation laser and layers configuration with cylinder radius in the distance varations.

As expressed in eq.(3), the $\mathrm{I}^{b}$ is depends on the extinction coefficient, angle and layers depth counter. Our numerical calculations show that $\mathrm{I}^{b}$ in the skin tissue is much greater than water medium, due to differences in the refractive index, the Rayleigh length, beam radius and $I_{0}(z)$ calculated. There are not differences of viewpoint $\mathrm{I}^{b}$ amount between layers configuration for skin tissue in the 2-D and 3-D models. The multi photon and cascade ionizations are the primary mechanisms for the USP laser ablation on the model skin tissue [14]. The chromophore ionization it should be pointed out that in the presented are calculations, the properties of the chromophores are assumed to be the same as the melanosome [14]. A USP laser system at wavelengths $532,580,1064 \mathrm{~nm}$ is considered. The free-electron density was estimated in the orders of $10^{-40}-10^{27} \mathrm{~cm}^{-3}$ We assumed average laser power is $0.065 \mathrm{~W}$ and cylinderradius is $(\mathrm{r}=-12.5 \ldots 12.5 \mu \mathrm{m})$. For chromophore ionization with the picosecond and the femtosecond pulses, at all the wavelengths 532, 580 and $1064 \mathrm{~nm}$, from our calculations results we found that the good agreements between angle and the layer depth and the distance of focal spot. For example, according to our calculations for skin tissue show that maximum of generated chromophoreionization at three wavelength for dermis layer at angles $16^{\circ}$ and $22^{\circ}$ is more than for epidermis layer. As expressed in (9)-(12), the multi photon ionization rate is proportional to $G^{K}$ and the chromophore ionization rate depends on the absorption of laser pulse energy $F(t) \mu_{a}$ [14]. The chromophore ionization does not contribute too much to the generation of the seed electrons because of the relatively weak absorption of the chromophores $\left(\sigma_{a}=1.85 \mathrm{~mm}^{-1}\right)$ at mentionedwavelengths[14]. Therefore, in the following calculations concerning the chromophore ionization in skin tissues induced by thepicosecond and femtosecond lasers. Figure 3 to 8 , show that the variations of choromophore ionization in terms of time for skin tissue at certain physical condition .According to our calculations the maximum of generated chromophore ionization at the wavelength $532 \mathrm{~nm}$ with $6000 \mathrm{ps}$ pulse duration of layer dermis angle $16^{\circ}$ at the time of $2.38 \times 10^{-8} \mathrm{~s}$ is $8.09 \times 10^{33} \mathrm{~cm}^{-3}$, for skin tissue. 


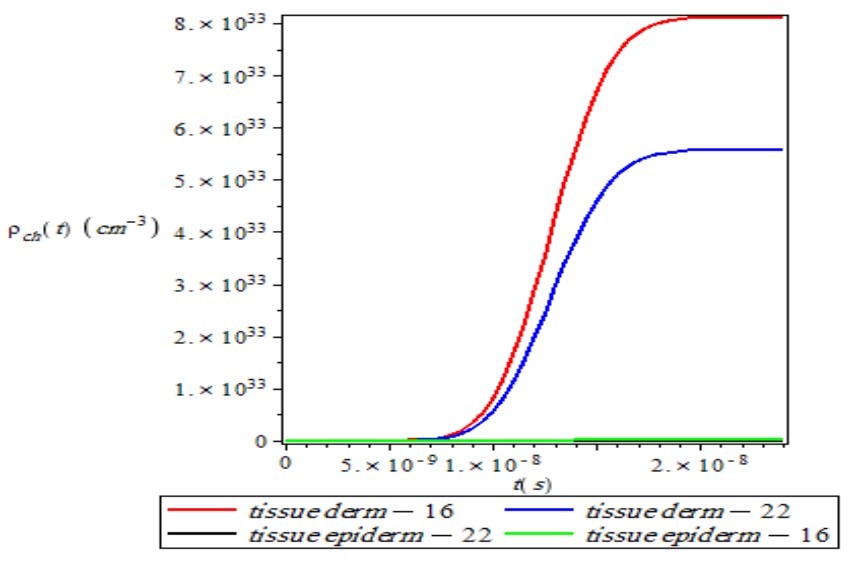

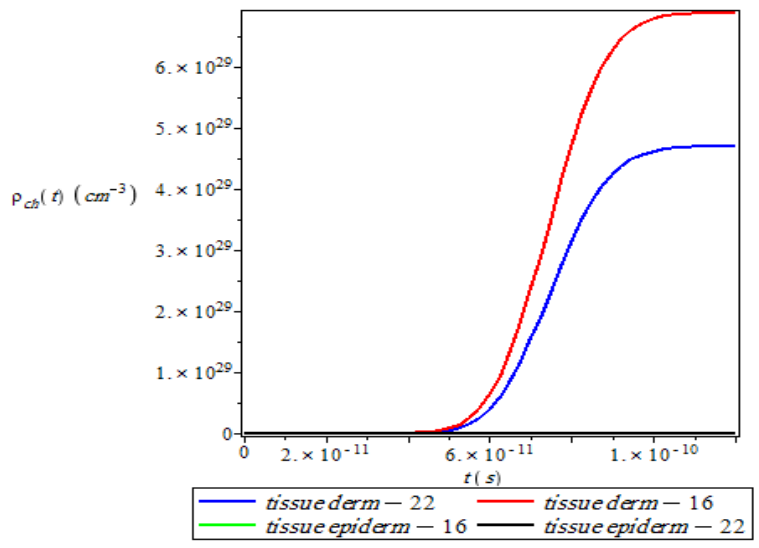

b

Figure 8. Comparison of the choromophore ionization as a function of the time in the wavelength $532 \mathrm{~nm}$ at two angles $16^{\circ}, 22^{\circ}$ for dermis and epidermis layers a) $2 \omega_{0 R}=5.3 \mu \mathrm{m}$ a) $2 \omega_{0 R}=3.4 \mu \mathrm{m}$ in the skin tissue.

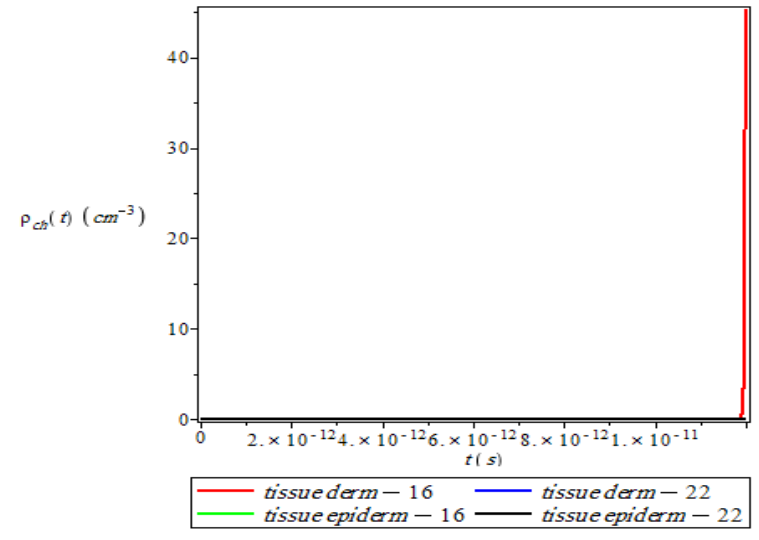

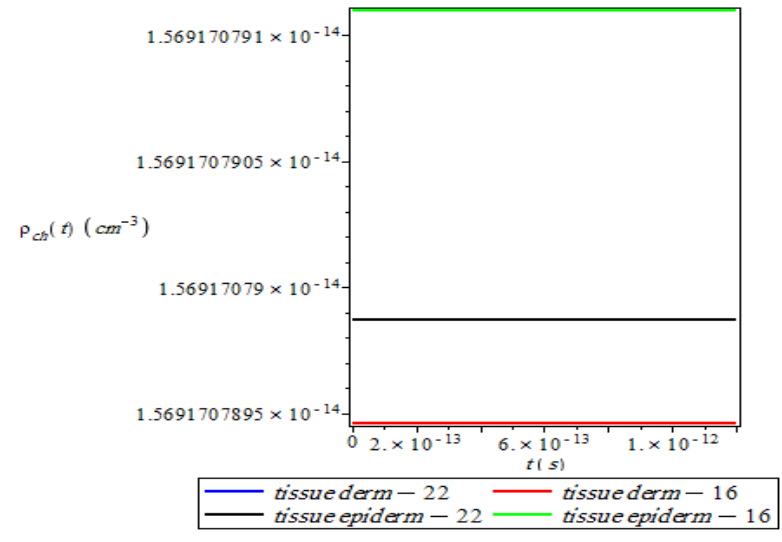

$\mathrm{b}$

Figure 9. Comparison of the chromophore ionization as a function of the time in the wavelength $580 \mathrm{~nm}$ at two angles $16^{\circ}, 22^{\circ}$ for dermis and epidermis layersa) $\left.2 \omega_{0 R}=5 \mu \mathrm{mb}\right) 2 \omega_{0 R}=5 \mu \mathrm{m}$ and $t_{p}=0.3 \mathrm{ps}$ in the skin tissue.

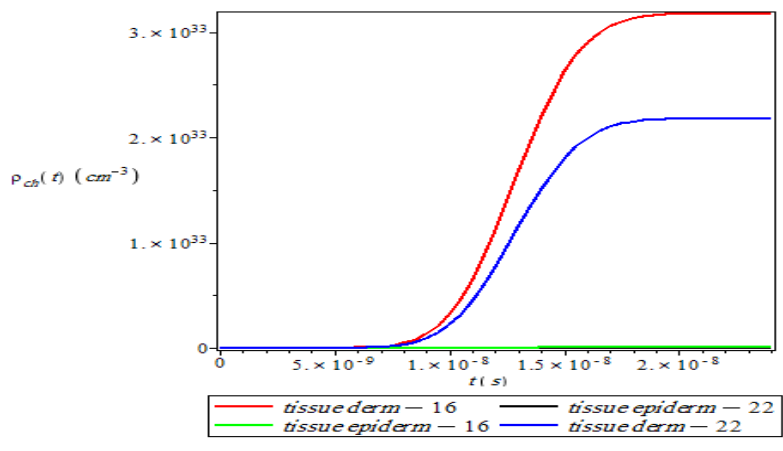

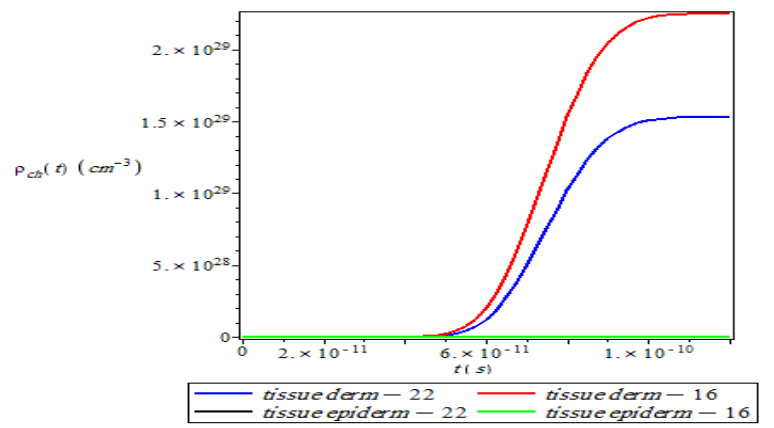

Figure 10. Comparison of the chromophore ionization as a function of the time in the wavelength 1064 nm at two angles $16^{\circ}, 22^{\circ}$ for dermis and epidermis layers $a) 2 \omega_{0 R}=7.7 \mu \mathrm{m}$ and $\left.t_{p}=6000 \mathrm{psb}\right) 2 \omega_{0 R}=4.7 \mu \mathrm{m}$ and $t_{p}=30 \mathrm{psin}$ the skin tissue.

The minimum of generated chromophor ionization at the 3 ps pulse at wavelength $580 \mathrm{~nm}$ and angle $22^{\circ}$ of epidermis layer at the time of $1.19 \times 10^{-11}$ sis $3.17 \times$ $10^{-14} \mathrm{~cm}^{-3}$ for skin tissue. Figure 9 shows chromophore ionization in skin tissue with the $0.3 \mathrm{ps}$ and 0.1 pulses the layers configuration with considered amount, angle, pulse duration and the time integral of the incident radiation and distance of focal spot. The layers configuration duo to generatedchromophor ionization at the different pulses and wavelengths are, dermis $16^{\circ}$, dermis $22^{\circ}$, epidermis $16^{\circ}$ and 
epidermis $22^{\circ}$,respectively. There are not differences of the viewpoint amount chromophore ionization in the layers configuration between for each medium in the 2-D and 3-D models, but from results of our calculations we found that differences are between layers configuration in the chromophore ionization with free electron density at skin

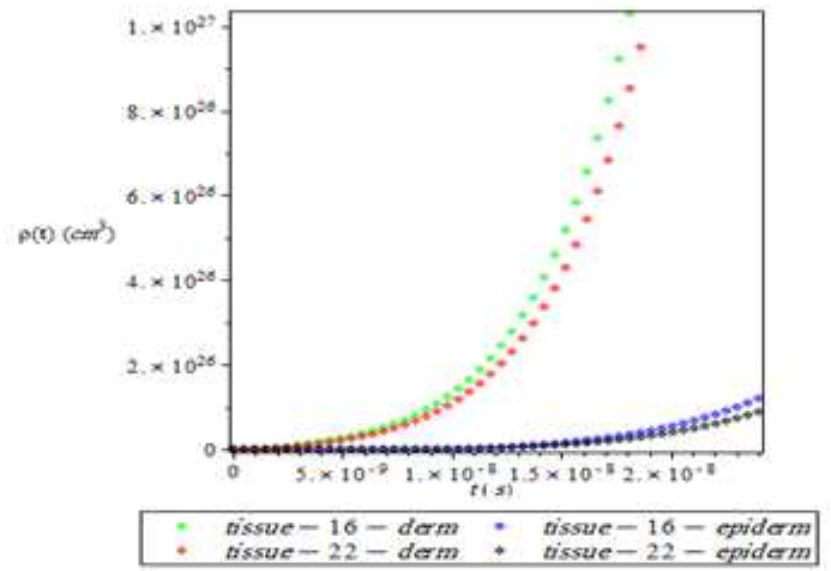

tissue these differences are due to choose the Runge-Kutta algorithm and critical electron density. Also, in fig 8 to 10 , we see that the two dimensional diagrams of time dependent free electron density for different wavelengths at various physical conditions for skin tissue (epidermis and dermis) at choosing the incident angles $16^{\circ}$ and $22^{\circ}$.

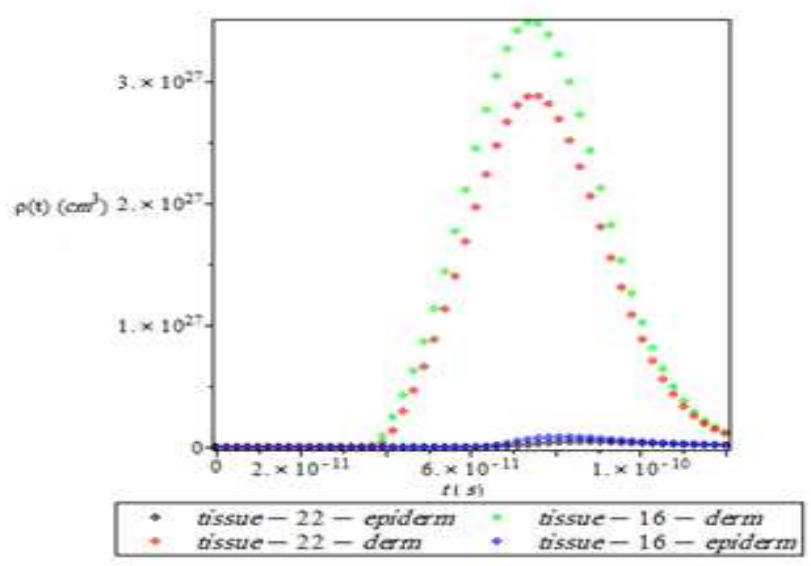

$\mathrm{b}$

Figure 11. Comparison of the free electron density as a function of the time in the wavelength $532 \mathrm{~nm}$ a) $2 \omega_{0 R}=5.3 \mu \mathrm{m}$ andt $\left.t_{p}=6000 \mathrm{psb}\right) 2 \omega_{0 R}=$ $3.4 \mu \mathrm{m}$ and $t_{p}=30 \mathrm{psin}$ the skin tissue.

Table 3. Comparison of the peak spot the free electron density in the skin tissue for wavelength $532 \mathrm{~nm}$ with $2 \omega_{0 R}=5.3 \mu \mathrm{m}$ and $t_{p}=6000 \mathrm{ps}$ at two angles $16^{\circ}, 22^{\circ}$ for dermis and epidermis layers.

\begin{tabular}{cccc}
\hline Tissue type & $\boldsymbol{\rho}_{\boldsymbol{s}}(\boldsymbol{t}) \mathbf{c m}^{-3}$ & $\boldsymbol{\rho}_{\boldsymbol{c h s}}(\boldsymbol{t}) \mathbf{c m}^{-3}$ & $\boldsymbol{I}_{\boldsymbol{s}}^{\boldsymbol{b}}(\boldsymbol{r}, \boldsymbol{z}, \boldsymbol{t})\left(\frac{\boldsymbol{w}}{\mathbf{c m}^{\mathbf{2}}}\right)$ \\
\hline $\begin{array}{c}\text { Dermis-angle } \\
16\end{array}$ & $8.25 \times 10^{26}$ & $8.09 \times 10^{33}$ & 68063.12 \\
$\begin{array}{c}\text { Epidermis- } \\
\text { angle16 }\end{array}$ & $1.22 \times 10^{26}$ & $1.08 \times 10^{31}$ & 2069.02 \\
$\begin{array}{c}\text { Dermis- } \\
\text { angle22 }\end{array}$ & $7.46 \times 10^{26}$ & $5.55 \times 10^{33}$ & 52981.49 \\
$\begin{array}{c}\text { epidermis- } \\
\text { angle22 }\end{array}$ & $9.09 \times 10^{25}$ & $5.91 \times 10^{30}$ & 1387.65 \\
\hline
\end{tabular}

Table 4. Comparison of the peak spot the free electron density in the skin tissue for wavelength $532 \mathrm{~nm}$ with $2 \omega_{0 R}=3.4 \mu \mathrm{m}$ and $t_{p}=30 \mathrm{ps}$ at two angles $16^{\circ}, 22^{\circ}$ for dermis and epidermis layers.

\begin{tabular}{cccc}
\hline Tissue type & $\boldsymbol{\rho}_{\boldsymbol{s}}(\boldsymbol{t}) \mathbf{c m}^{-3}$ & $\boldsymbol{\rho}_{\boldsymbol{c h s}}(\boldsymbol{t}) \boldsymbol{c m}^{-3}$ & $\boldsymbol{I}_{\boldsymbol{s}}^{\boldsymbol{b}}(\boldsymbol{r}, \boldsymbol{z}, \boldsymbol{t})\left(\frac{\boldsymbol{w}}{\mathbf{c m}^{\mathbf{2}}}\right)$ \\
\hline Dermis-angle 16 & $3.48 \times 10^{27}$ & $6.68 \times 10^{29}$ & 26464.4 \\
& & & \\
epidermisangle16 & $8.75 \times 10^{25}$ & $3.67 \times 10^{26}$ & 772.5 \\
& & & \\
Dermis-angle22 & $2.87 \times 10^{27}$ & $4.68 \times 10^{29}$ & 20475.96 \\
& & & \\
Epidermis- & $4.85 \times 10^{25}$ & $1.3 \times 10^{26}$ & 514.07 \\
\hline
\end{tabular}

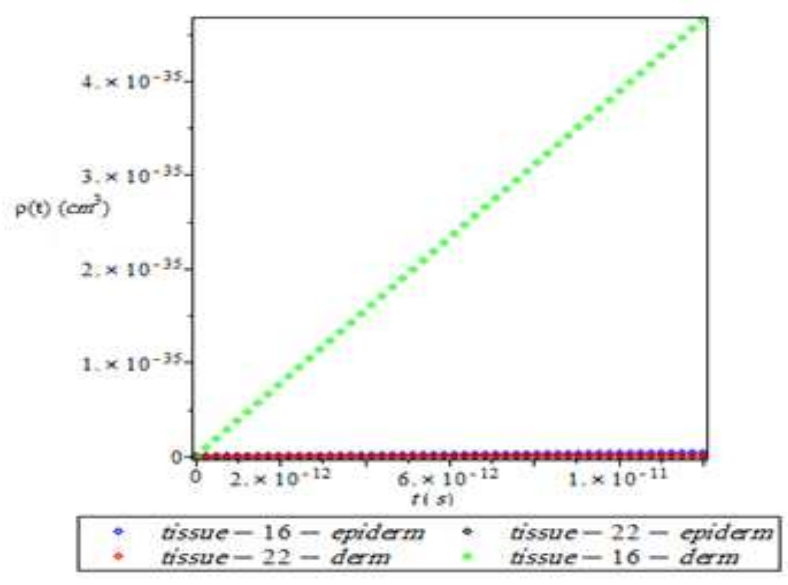

Figure 12. Comparison of the free electron density as a function of the time in the wavelength $580 \mathrm{~nm}$ at two angles $16^{\circ}, 22^{\circ}$ for dermis and epidermis layers a) $2 \omega_{0 R}=5 \mu m$ and $t_{p}=3 p s$.

Table 5. Comparison of the peak spot the free electron density in the skin tissue for wavelength $580 \mathrm{~nm}$ with $2 \omega_{0 R}=5 \mu \mathrm{m}, t_{p}=3 p$ sat two angles $16^{\circ}, 22^{\circ}$ for dermis and epidermis layers.

\begin{tabular}{cccc}
\hline Tissue type & $\boldsymbol{\rho}_{\boldsymbol{s}}(\boldsymbol{t}) \mathbf{c m}^{-3}$ & $\boldsymbol{\rho}_{\boldsymbol{c h s}}(\boldsymbol{t}) \mathbf{c m}^{-3}$ & $\boldsymbol{I}_{\boldsymbol{s}}^{\boldsymbol{b}}(\boldsymbol{r}, \boldsymbol{z}, \boldsymbol{t})\left(\frac{\boldsymbol{w}}{\boldsymbol{c m}^{2}}\right)$ \\
\hline $\begin{array}{c}\text { Dermis-angle } \\
16\end{array}$ & $4.46 \times 10^{-35}$ & 45.2 & 191.25 \\
$\begin{array}{c}\text { epidermis- } \\
\text { angle16 }\end{array}$ & $4.32 \times 10^{-37}$ & $3.17 \times 10^{-14}$ & 5.741 \\
$\begin{array}{c}\text { Dermisangle22 } \\
\text { epidermis- } \\
\text { angle22 }\end{array}$ & $7.92 \times 10^{-35}$ & $7.9 \times 10^{-10}$ & 43.67 \\
\hline
\end{tabular}




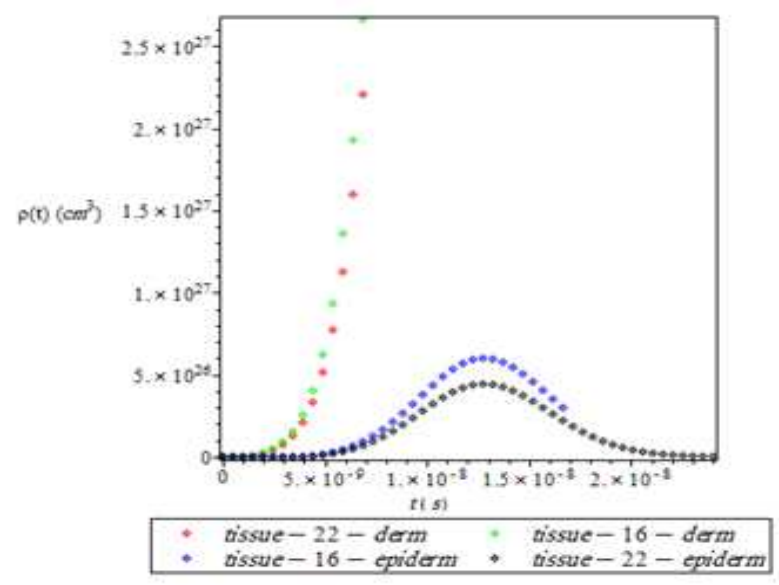

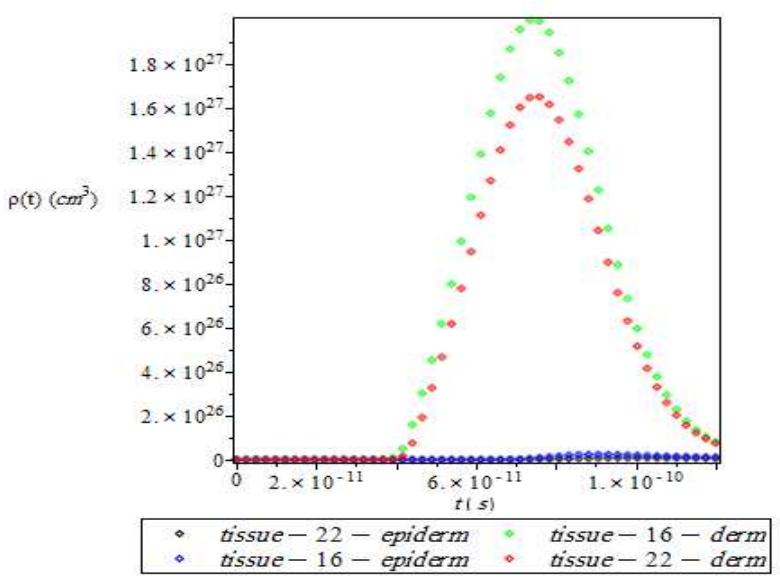

b

Figure 13. Comparison of the free electron density as a function of the time in the wavelength $1064 \mathrm{~nm}$ at two angles $16^{\circ}, 22^{\circ}$ for dermis and epidermis layers $a) 2 \omega_{0 R}=7.7 \mu \mathrm{m}$ and $\left.t_{p}=6000 \mathrm{psb}\right) 2 \omega_{0 R}=4.7 \mu \mathrm{m}$ andt $t_{p}=30 \mathrm{psin}$ the skin tissue.

Table 6. Comparison of the peak spot the free electron density in the skin tissue for wavelength $1064 \mathrm{~nm}$ with $2 \omega_{0 R}=7.7 \mu \mathrm{m}$ and $t_{p}=6000 \mathrm{psat}$ two angles $16^{\circ}, 22^{\circ}$ for dermis and epidermis layers.

\begin{tabular}{cccc}
\hline Tissue type & $\boldsymbol{\rho}_{\boldsymbol{s}}(\boldsymbol{t}) \mathbf{c m}^{-\mathbf{3}}$ & $\boldsymbol{\rho}_{\boldsymbol{c h s}}(\boldsymbol{t}) \mathbf{c m}^{-\mathbf{3}}$ & $\boldsymbol{I}_{\boldsymbol{s}}^{\boldsymbol{b}}(\boldsymbol{r}, \mathbf{z}, \boldsymbol{t})\left(\frac{\boldsymbol{W}}{\mathbf{c m}^{2}}\right)$ \\
\hline $\begin{array}{c}\text { Dermis-angle } \\
16\end{array}$ & $1.92 \times 10^{27}$ & $3.17 \times 10^{33}$ & 36540.05 \\
$\begin{array}{c}\text { epidermis- } \\
\text { angle16 }\end{array}$ & $6.1 \times 10^{26}$ & $4.26 \times 10^{30}$ & 1111.54 \\
$\begin{array}{c}\text { Dermis- } \\
\text { angle22 }\end{array}$ & $2.2 \times 10^{27}$ & $2.18 \times 10^{33}$ & 28362.54 \\
$\begin{array}{c}\text { epidermis- } \\
\text { angle22 }\end{array}$ & $4.54 \times 10^{26}$ & $2.33 \times 10^{30}$ & 743.49 \\
\hline
\end{tabular}

Table 7. Comparison of the peak spot the free electron density in the skin tissue for wavelength $1064 \mathrm{~nm}$ with $2 \omega_{0 R}=4.7 \mu \mathrm{m}$ and $t_{p}=30$ psat two angles $16^{\circ}, 22^{\circ}$ for dermis and epidermis layers.

\begin{tabular}{cccc}
\hline Tissue type & $\boldsymbol{\rho}_{\boldsymbol{s}}(\boldsymbol{t}) \mathbf{c m}^{-3}$ & $\boldsymbol{\rho}_{\boldsymbol{c h s}}(\boldsymbol{t}) \mathbf{c m}^{-3}$ & $\boldsymbol{I}_{\boldsymbol{s}}^{\boldsymbol{b}}(\boldsymbol{r}, \mathbf{z}, \boldsymbol{t})\left(\frac{\boldsymbol{W}}{\mathbf{c m}^{2}}\right)$ \\
\hline $\begin{array}{c}\text { Dermis-angle } \\
16\end{array}$ & $1.99 \times 10^{27}$ & $2.25 \times 10^{29}$ & 12662.7 \\
$\begin{array}{c}\text { epidermis- } \\
\text { angle16 }\end{array}$ & $2.57 \times 10^{25}$ & $4.78 \times 10^{25}$ & 369.13 \\
$\begin{array}{c}\text { Dermis- } \\
\text { angle22 } \\
\text { epidermis- } \\
\text { angle22 }\end{array}$ & $1.65 \times 10^{27}$ & $1.53 \times 10^{29}$ & 9826.43 \\
\hline
\end{tabular}

In tables 3 to 7 , our calculations results of numerical values of intensity radiation laser, chromophor ionization and time dependent free electron density for various wavelengths at different physical conditions at two angles $16^{\circ}$ and $22^{\circ}$ for two layers (epidermis and dermis ) for skin tissue. By seeing the table 3 to 7 , we will find that the good agreement is seen between free electron density and critical electron density and layers configuration with considered wavelengths and pulse durations. Time dependent free electron density for wavelength of $1064 \mathrm{~nm}$, with $6 \mathrm{~ns}$ pulse duration is the slight difference with the other wavelengths at various physical conditions from the point view of layersconfiguration. According to our calculations the critical free electron density in skin tissue at the wavelengths $1064 \mathrm{~nm}$ with6000ps pulse duration of layer dermis $22^{\circ}$ is, $6.91 \times 10^{24} \mathrm{~cm}^{-3}$, and for layer dermis $16^{\circ}$ is zero. This difference is due to critical free electron density, therefor, according to our calculations, the maximum of generated free electron density at the dermis layer of skin tissue are $2.2 \times 10^{27}, 1.92 \times 10^{27} \mathrm{~cm}^{-3}$ for angle $22^{\circ}$ and $16^{\circ}$, respectively The maximum critical free-electron density in skin tissue at $532 \mathrm{~nm}$ of and with 30 ps pulse of dermis layer $22^{\circ}$ is, $1.09 \times 10^{25} \mathrm{~cm}^{-3}$ and minimum at 580 $\mathrm{nm}$ with 3 ps pulse of epidermis layer $22^{\circ}$ is $6 \times$ $10^{-40} \mathrm{~cm}^{-3}$. Our computions show that for ablation in skin tissue at $580 \mathrm{~nm}$ wavelength with two pulse duration $0.1 \mathrm{ps}$ and 0.3 ps the time dependent free electron density is about 0 . This is duo to very short pulse duration and $I^{b} \cong 0$. Our calculations show that free electron density and chromophore ionization is increasing with increasing time and the time dependent integral of the incident radiation and the radiation intensity.

For ablation with the picosecond and femtosecond pulses, at all the wavelength 532, 580 and $1064 \mathrm{~nm}$, from calculations results we conclude that the good agreement between layers configuration with considering angle andthe distance of spot focal. Therefore from table 3 to 7 we see that concerning the ablation in skin tissue induced by the picosecond and femtosecond lasers, maximum free electron density for wavelength 532 with 30 ps pulse duration of dermis layer at angle $16^{\circ}$ is $3.48 \times 10^{27} \mathrm{~cm}^{-3}$ in the time $7.25 \times 10^{-11} \mathrm{~s}$ for skin tissue. Therefor, according to our calculations the minimum of generated free electron density of epidermis $22^{\circ}$ at 3 ps pulse and $580 \mathrm{~nm}$ wavelength at the time of $1.19 \times 10^{-11}$ sis $6 \times 10^{-40} \mathrm{~cm}^{-3}$, for skin tissue. From fig (11)-(13) we see that the free electron density could expand initially of the occurrence of cascade ionization with more laser energy. Focused laser beam was incident on the surface of the epidermis or inside the skin tissue. A USP laser system at wavelength $1064 \mathrm{~nm}$ 
with 30 ps pulse duration is considered. The critical freeelectron density would be estimated in the order of $10^{22}-10^{25} \mathrm{~cm}^{-3}$. To remove the cancerous cells exiting in the skin layers effectively, a laser beam can be focused inside the skin to the dermis angle 16 degree surface. The critical electron density epidermis layer is small therefore, the free - electron density is a very small in a dermis layer.

\section{Conclusions}

In this work, our studies are established on the ultra short pulsed laser on the skin in cylindrical coordinates. The temporal evolutions of the free-electron density are calculated using a numerical methods(fourth-order RungeKutta). A parametric study with regarding to the free electron density (plasma) in the skin tissue on the dermis and epidermis layers at angle 16 and 22 degree are concluded. In this work, a 2-D and 3-D models are established in combining the chromophore ionization rate and the $\mathrm{I}^{b}$ to investigate the temporal evolutions of the freeelectron density in skin tissues. From our obtaing results we found that the good agreement between layers configuration with considering angle, the extinction coefficient, layers depth and the distance of spot focal. Our studies on this work, show that if the amount of radiation intensity of pulsed laser is increased and the distance of focal spot is decreased, the amount of time dependent freeelectron density more and more, such that this case is proper for skin cancer treatment. Also, our calculations for skin tissue show that optimum time dependent free electron density, $\mathrm{I}^{b}$ and the chromophore ionization rate for dermis layer at angle $16^{\circ}$ and $22^{\circ}$ are functions of wavelength, beam width, beam radius, amplitude of the beam radiation strength. Also pulse duration is more than for epidermis layer. Our calculations show that free electron density and chromophore ionization is increasing with increasing time and the time dependentintegral of the incident radiation and the radiation intensity. From calculations results we found that the good agreement between layers configuration of viewpoint amount for the free electron density rate equation and chromophore ionization with considering angle and distance of focal spot and $\mathrm{I}^{b}$. The first layer is dermis with angle 16 degree, latter layer is dermis with 22 degree, the $3^{\text {rd }}$ layer is epidermis 16 degree and the last layer is epidermis 22 degree. The influence of various ionization mechanism including, chromophore ionization pathways on the process of plasma generation for the model skin tissue is investigated. A good agreement is found between layers configuration in 2-D and 3-D models of choromophor ionization in skin tissue but the slight differences are between layers configuration in chromophore ionization with free electron density at skin tissue can due to differences in the Runge-Kutta algorithm and critical electron density.

\section{References}

[1] Y.R. Shen, The Principles of Nonlinear Optics (Wiley, New York, 1984)

[2] J. Kasparian, J. Solle, M. Richard, J.-P. Wolf, Appl. Phys. B 79,947 (2004)

[3] K.Köning, I.Riemann,P.Fischer,K.Halbhuber,Cell.Mol.biol.45,195(199 9)

[4] K.Schutze,G.Lahr,Nat.Biotechnol.16,737(1998)

[5] J. Noack, A. Vogel, IEEE J. Quantum Electron. 35, 1156 (1999)

[6] M.H. Niemz, E.G. Klancnik, J.F. Bille, Lasers Surg. Med. $11,426(1991)$

[7] F.Docchio,P.Regondi,M.R.c.Capon,J.Mellerio,appl.Opt.27,3 661(1988)

[8] F.Docchio,P.Regondi,M.R.c.Capon,J.Mellerio,appl.Opt.27,3 669(1988)

[9] C.H .Fan, J. Sun, J.P. Longtin, J. Heat Transf.124,275(2002)

[10] J. Zhou, J.H. Chen, Y. Zhang, Appl. Phys. B 90,141(2008)

[11] H.Huang,Z.Guo,J.Phys.D,Appl.Phys.42,165204(2009)

[12] H. Huang, Z. Guo, Lasers Med.Sci.25,517(2010)

[13] . J.Jiao,Z.Guo,Phys.Med.Biol.54,4225(2009)

[14] J. Jiao, Z. Guo, Appl Phys,195,(2011)

[15] H.Huang,Z.Guo,J.Phys.D,Appl.Phys.42,165204(2009)

[16] H. Huang, Z. Guo, Lasers Med.Sci.25,517(2010)

[17] J.Jiao,Z.Guo,Phys.Med.Biol.54,4225(2009)

[18] P. Kennedy, IEEE J. Quantum Electron.31,2241(1995)

[19] F.William,S.p.Varma,S.Hillenius,J.Chem.Phys.64,1549(197 6)

[20] Q. Y. Fang, X. H. Hu, IEEE j,QuantumElectron.40,69(2004)

[21] H.Ding,J.Q.Lu,W.a.Wooden,P.J.Kragel,X.H.Hu,Phys.Med.B io. $51,1479(2006)$ 